ORIGINAL ARTICLE

\title{
Longitudinal relation between limited joint mobility, height, insulin-like growth factor 1 levels, and risk of developing microalbuminuria: the Oxford Regional Prospective Study
}

\author{
R Amin, T K Bahu, B Widmer, R N Dalton, D B Dunger
}

Arch Dis Child 2005;90:1039-1044. doi: 10.1136/adc.2004.067272

See end of article for authors' affiliations

Correspondence to:

Prof. D B Dunger,

University Department of

Paediatrics, Box 116, Level

8, Addenbrookes Hospital,

Hills Road, Cambridge

CB2 2QQ, UK; dbd25@

cam.ac.uk

Accepted 6 April 2005

\begin{abstract}
Aims: To determine risk factors for development of microalbuminuria (MA) in relation to detection of limited joint mobility ( $\triangle M+)$ of the interphalangeal joints in a longitudinal cohort of type 1 diabetic (TIDM) subjects.

Methods: A total of 479 TIDM subjects diagnosed $<16$ years were followed from diagnosis of diabetes with annual assessments consisting of assessment of $\mathrm{LM}$, measurement of $\mathrm{HbAl}_{c}$ and insulin-like growth factor 1 (IGF-1), and three urine samples for albumin:creatinine ratio (ACR).

Results: After a median follow up of 10.9 years, 162 subjects (35.1\%) developed LM at median age 13.0 years and duration 5.2 years. More subjects developed LM after compared to before puberty $167.6 \mathrm{v}$ 32.4\%). In LM+ compared to LM- subjects, HbAlc (mean 10.1 (SD 1.6) v $9.6(1.4) \%)$ ) and ACR levels (median 1.1 (range 0.2-242.9) v $0.9(0.4-70.7) \mathrm{mg} / \mathrm{mmol}$ ) were higher, and in a Cox model probability of developing $\mathrm{LM}$ was related to puberty and higher $\mathrm{HbAlc}$ levels. ACR levels were higher after detection of LM compared to before (median 1.2 (range $0.4-102.6) \vee 0.8(0.2-181.9) \mathrm{mg} / \mathrm{mmol}$ ). Probability of developing MA was related to puberty, HbAlc, female sex, and presence of $\mathrm{LM}$ (a 1.9-fold increased risk). Both LM and MA were associated with lower height SDS (LM: mean 0.0 (SD 1.0) v 0.2 (1.1); MA: $0.0(1.0) \vee 0.2$ (SD 1.0)) and lower IGF-1 levels.

Conclusion: The development of $\mathrm{DM}$ was associated with an increased risk of microalbuminuria, independent of glycaemic control. Risk for both microalbuminuria and LM was associated with puberty, reduced growth, and reduced IGF-1 levels, and may indicate underlying shared pathogenic mechanisms.
\end{abstract}

l type 1 diabetes mellitus (TIDM) prevalence of limited joint mobility (LJM), as shown by the inability of full digital extension, is increased. ${ }^{1-6}$ Studies show prevalence of LJM relates to poor glycaemic control, age, diabetes duration, and puberty, but data are inconsistent. ${ }^{1-4}$ 7-13

The development of LJM may be associated with an increased prevalence of microvascular complications ${ }^{26}{ }^{10} 14-16$ and may be an early marker of these complications. Three surveys show correlation between development of LJM and retinopathy, ${ }^{314} 15$ and similar associations have been described with microalbuminuria (MA) ${ }^{3} 1014$ and hypertension; ${ }^{10}$ however these studies are largely cross-sectional. ${ }^{1} 247-11$ 17 Furthermore results may be confounded by age, duration of follow up, puberty, and differing definitions of LJM, and other studies have failed to confirm the original observations. ${ }^{481317} \mathrm{Few}$ prospective data exist. Rosenbloom et al have shown longitudinal association between LJM, glycaemic control, ${ }^{12}$ and microvascular disease, ${ }^{6}$ with the presence of LJM predictive of early retinopathy. ${ }^{18}$

The risk factors for development of LJM may be similar to those that are associated with microvascular disease, thus the same pathogenic mechanisms may be involved. ${ }^{6}$ Studies show a relation between LJM and attenuated growth, ${ }^{4} 8919$ and this is also reported in relation to the development of $\mathrm{MA}^{20-22}$ retinopathy, ${ }^{23}$ and mortality in TIDM subjects. ${ }^{24}$ These associations may relate to reduced insulin-like growth factor 1 (IGF-1) levels which have been implicated in the development of $\mathrm{MA}^{25}$ and retinopathy. ${ }^{26}$

These associations have yet to be explored in large, population based, prospective studies. The Oxford Regional Prospective Study (ORPS) is a regionally defined, natural history study following children with TIDM longitudinally from diagnosis of diabetes, through puberty into young adulthood. ${ }^{25}$ We describe risk factors for developing LJM and explore the relation between MA, growth, IGF-1 levels, and LJM.

\section{METHODS \\ The ORPS cohort}

The ORPS was established in 1986 and the characteristics of the cohort have previously been described. ${ }^{27}$ Children $<16$ years in the region of Oxford Health Authority were recruited within three months of diagnosis of diabetes to receive annual assessments. Case ascertainment was $>95 \%$ with 479 subjects recruited into the study by 1997. Ethical approval was obtained from the district ethics committees in the region, with written consent from the parents. Children were asked to assent before entering the study. To date median duration of follow up is 10.9 years (range 6.0-17.8).

\section{Annual assessments}

Subjects were assessed annually from the first year of diagnosis for height, weight, blood pressure (BP), and three consecutive early morning (first void) urine specimens for the measurement of albumin:creatinine ratio (ACR). Blood samples were collected for the centralised measurement of $\mathrm{HbAl}_{\mathrm{c}}$ and insulin-like growth factor $\mathrm{l}$ (IGF-1). At each assessment LJM was assessed by approximation of the palmar surfaces of both hands actively and passively, with fingers fanned and wrists extended. Parents were measured

Abbreviations: $A C R$, albumin:creatinine ratio; $B P$, blood pressure; $C V$, coefficient of variation; GH, growth hormone; IGF-1, insulin-like growth factor 1; LM, limited joint mobility; MA, microalbuminuria; ORPS, Oxford Regional Prospective Study; SDS, standard deviation score; TIDM, type 1 diabetes mellitus 


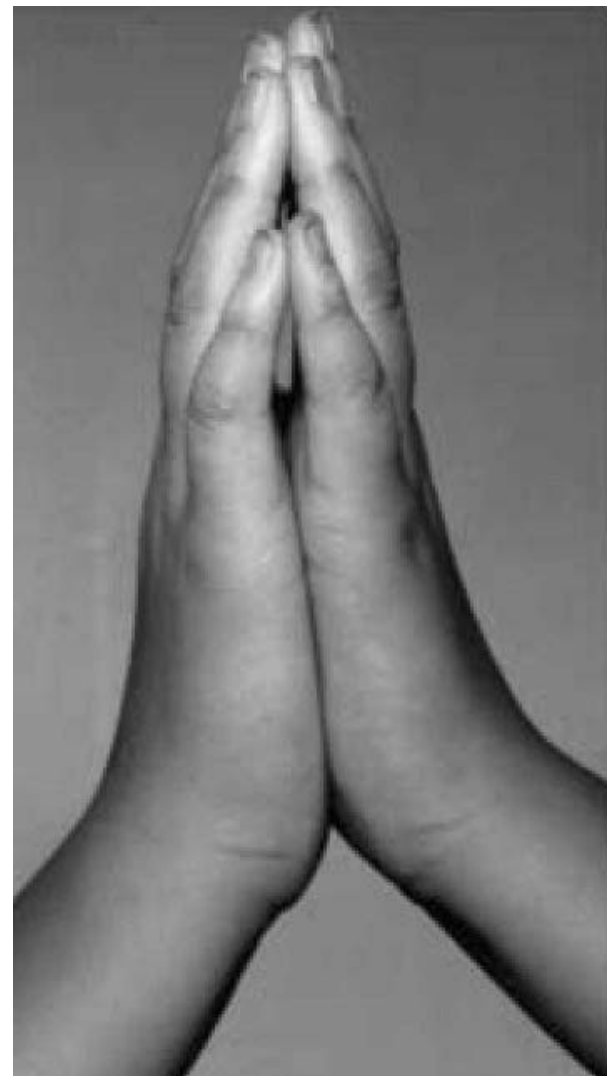

Figure 1 Child with TIDM undertaking the prayer sign, showing mild LM. Consent was obtained for publication of this figure.

for height, weight, and BP, and examined for presence of LJM. In each district examinations were undertaken by the same observer, who was blind to the results of previous years.

\section{Definition of LJM and microalbuminuria}

Joint limitation was classified as: normal (no joint limitation or limitation of two or more proximal inter- or metacarpophalangeal joints at one annual assessment only); mild (limitation of two proximal inter- or metacarpo-phalangeal joints on two or more annual assessments) (fig 1); moderate (limitation of three or more inter- or metacarpo-phalangeal joints on two or more annual assessments); and severe (obvious hand deformity at rest). Thus for the purposes of this study, LJM was diagnosed only if present for two or more years. This strict definition of LJM was used to the reduce intra-observer variation in diagnosis that may occur in a longitudinal study. Subjects with mild, moderate, and severe LJM were designated as cases (LJM+) and the remainder were designated as LJM- controls. Although no non-diabetic controls were available for comparison in the current study, previous studies have confirmed the reliability of using this definition, yielding consistent and reproducible results by different observers, compared to non-diabetic subjects. ${ }^{3} 9^{1012}$ LJM tends to be painless, asymptomatic, and not disabling unless severe, when it is often associated with large joint involvement. ${ }^{9}$

MA was defined as an ACR $>3.5 \mathrm{mg} / \mathrm{mmol}$ in males and $>4.0 \mathrm{mg} / \mathrm{mmol}$ in females, and $<35 \mathrm{mg} / \mathrm{mmol}$ in two of three consecutive early morning urine collections. ${ }^{25}$ Consistent with previous ORPS studies, ${ }^{27}$ persistent MA was defined as an ACR within the microalbuminuric range for two or more consecutive years. Transient MA was defined as microalbuminuria for one year, then regression to normoalbuminuria the subsequent year.

\section{Methods}

Auxology

Height was measured on wall mounted stadiometers, weight measured on electronic scales.

\section{Albumin assay}

Until 1994 urine samples were stored at $-20^{\circ} \mathrm{C}$ and after this time at $-70^{\circ} \mathrm{C}$. Albumin was measured centrally by a double antibody ELISA method. ${ }^{27}$ The within and in-between assay coefficient of variation (CV) was $6 \%$ and $12 \%$ respectively.

\section{Creatinine}

Creatinine was measured using a modified Jaffe method (Unimate 7, Roche Diagnostic Systems, Basel, Switzerland) on a Cobas Mira (Roche Diagnostic Systems, Basel, Switzerland) automated spectrophotometer. The CV was $2 \%$ at $2.2 \mathrm{mmol} / \mathrm{l}$.

$\mathrm{HbA} 1_{\mathrm{c}}$

Glycated haemoglobin was measured initially by an electrophoretic method (Ciba Corning Diagnostics, Halstead, UK) and then by high performance liquid chromatography (HPLC-DIAMAT; Bio-Rad, Hemel Hempstaed, UK). ${ }^{28}$ The relation between the two methods was carefully evaluated and has been described previously. ${ }^{27}$ The within batch CV for the HPLC method was $2.2 \%$ and $1.3 \%$ at a level of $9.8 \%$ and $10.1 \%$ respectively. The between batch CV was $3.5 \%$ and $2.2 \%$ at $5.6 \%$ and $10.1 \%$ respectively.

\section{IGF-1}

IGF-1 levels were determined by radioimmunoassay following acid-acetone extraction using rabbit anti-serum developed by L Underwood (North Carolina University, Chapel Hill). The assay was standardised against a pool of normal human serum, defined as containing $1.0 \mathrm{U}$ IGF-1/ml (equivalent to $159 \mathrm{ng} / \mathrm{ml}$ of a purified preparation of IGF$\mathrm{I}^{29}$ ). The intra-assay and inter-assay CV were $3.5 \%$ and $6.2 \%$ respectively.

\section{Statistical methods}

ACR was calculated from the geometric mean of the three annual urine samples. Height, and BMI standard deviation scores (SDS) were calculated from the UK growth reference charts. ${ }^{30}$ Data were normally distributed, except ACR which were log transformed to allow parametric analyses. For each subject data were summarised as means and compared using the independent $t$ test, $\chi^{2}$ analyses, a Kaplan-Meier survival curve, and log rank test. A Cox method was used to measure the proportional contribution of covariates to risk of developing LJM or MA. To display longitudinal changes in data, multilevel modelling software was used (MLwiN version 1.0 beta, Institute of Education, London, UK). This is an extension of multiple regression, using repeated measures data and analyses within and between individual effects, allowing consideration of individual curves and their summation by predefined groups. SPSS version 10.0 was used for analysis. Data are presented as mean (SD) or median (range). A $p$ value $<0.05$ was considered significant.

\section{RESULTS}

\section{Demographic characteristics of cohort}

From 479 subjects (262 males), after a median of six observations per subject (range 1-16), 178 subjects (37.2\%) met our definition for LJM. Sixteen subjects had evidence of LJM within one year of diagnosis of diabetes and were excluded from further analyses as it was assumed that this could be unrelated to the effects of diabetes. However, no differences existed in these subjects compared to the remainder of the cohort, in terms of glycaemic control and 
Table 1 Characteristics of cohort comparing all subjects with $(\mathrm{L} M+$ ) and without (LM-) limited joint mobility

\begin{tabular}{llll}
\hline & UM+ & UM- & p value \\
\hline Demographic characteristics & $162(87$ male) & $301(167$ male) & \\
Sample size & $8.5(0.4-15.9)$ & $9.6(0.4-15.6)$ & 0.03 \\
Age diagnosis of diabetes (years) & $8.6(4.2-15.0)$ & $8.0(4.1-15.7)$ & 0.1 \\
Duration of diabetes (years) & $3.3(0.6)$ & $3.3(0.6)$ & 0.8 \\
Birth weight (kg) & $47(29.0 \%)$ & $80(26.6 \%)$ & 0.9 \\
Smokers & $0.8(0.2)$ & $1.0(0.3)$ & 0.01 \\
Insulin dose (U/kg/day) & & & \\
Clinical and biochemical characteristics & $10.1(1.6)$ & $9.6(1.4)$ & $<0.001$ \\
HbAlc (\%) & $39(24.1 \%)$ & $56(18.6 \%)$ & 0.3 \\
Subjects with microalbuminuria & $1.1(0.2-242.9)$ & $0.9(0.4-70.7)$ & 0.004 \\
ACR (mg/mmol) & $2.0(0.1-533.9)$ & $1.8(0.1-184.9)$ & 0.04 \\
ACR in microallbuminuric subjects only & $0.1(1.1)$ & $0.3(1.0)$ & 0.002 \\
(mg/mmol) & $0.0(1.0)$ & $0.2(1.1)$ & 0.001 \\
Height SDS at 1st assessment & $-0.8(1.3)$ & $-0.5(1.4)$ & 0.06 \\
Height SDS & $104.1(8.5)$ & $103.8(10.1)$ & 0.7 \\
IGF-1 SDS & $-1.1(0.7)$ & $-1.1(0.7)$ & 0.7 \\
Systolic BP (mm Hg) & $66.9(6.6)$ & $67.5(6.8)$ & 0.2 \\
Systolic BP SDS & $1.1(0.6)$ & $1.1(0.6)$ & 0.2 \\
Diastolic BP (mm Hg) & & \\
Diastolic BP SDS & &
\end{tabular}

Data are expressed as means (SD) or median (range) for the total duration of follow up, unless stated otherwise $A C R$, urine albumin:creatinine ratio; SDS, standard deviation score.

prevalence of MA (data not shown). Remaining analyses were restricted tol62 subjects $(35.1 \%)$ with LJM (LJM+) and 301 subjects without LJM (LJM-). The median age when LJM was first detected was 13.0 years (range 5.4-26.6) reflecting a median duration of diabetes of 5.2 years (1.013.6). Fifty subjects $(32.4 \%)$ developed LJM before puberty compared to 112 subjects $(67.6 \%)$ after puberty $(\mathrm{p}<0.004)$. The details of the cohort are described in table 1 .

\section{Relation of LJM to glycaemic control}

Mean $\mathrm{HbAl}_{\mathrm{c}}$ levels were higher in those with compared to those without LJM (table 1). Cumulative probability of developing LJM was greater in subjects with median HbAlc values greater than compared to less than $10 \%$ (fig 2; log rank test $\mathrm{p}=0.006$ for duration of diabetes).

\section{Relation of LJM to parental LJM and gender}

In subjects with compared to without LJM, prevalence of one or both parents with LJM was not increased (data not shown). The prevalence of LJM was no different in males compared to females (85 $(34.7 \%) \vee 77(38.9 \%), \chi^{2}=1.9$, $\mathrm{p}=0.5)$. However, in females $\mathrm{HbAl}_{\mathrm{c}}$ levels were higher in those with LJM ( 10.4 (SD 14) \% v 9.4 (SD 1.5) \%, p < 0.001 ), whereas in males no differences were found (9.9 (SD 1.4) $v$ 9.7 (SD 1.4), $\mathrm{p}=0.3$ ).

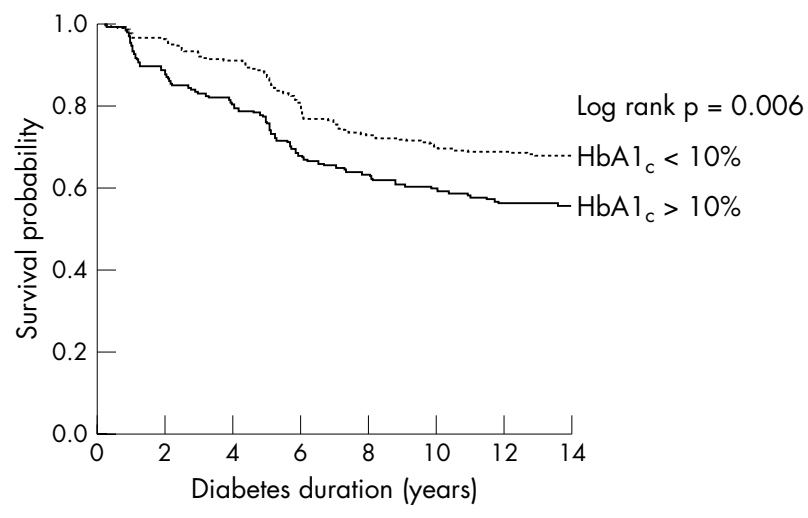

Figure 2 Kaplan-Meier survival curve for probability of developing limited joint mobility.

\section{Relation of LJM to growth and IGF-1 levels}

In subjects with LJM compared to those without LJM, height SDS at first assessment and mean lifetime height SDS were lower (table 1, fig 3A). Mean IGF-1 levels were also lower in the LJM+ group (182.6 (SD 63.6) v 199.8 (SD 79.5) ng/ml, $\mathrm{p}=0.04$ ).

\section{Determinants of risk for development of LJM using Cox method}

The probability of developing LJM (with duration of diabetes to onset of LJM as the time variable and puberty as the time dependent covariate) was related to puberty (a 1.4-fold increased risk with pubertal onset) and $\operatorname{HbAl}_{\mathrm{c}}$ (a $10 \%$ increased risk for a $1 \% \mathrm{HbAlc}$ rise), but not other covariates (table 2).

\section{Relation between LJM, IGF- 1 levels, height, and risk of microalbuminuria}

From 479 subjects in the cohort, 95 (19.8\%) developed microalbuminuria $(\mathrm{MA}+)$. For $\mathrm{MA}+$ subjects, the median age of onset was 15.4 years (range 6.0-24.4), and median duration of diabetes to first appearance of MA was 5.9 years (range 0.0-12.2). More subjects developed MA after puberty (age $>11$ years) compared to before puberty $(\mathrm{n}=83(87.2 \%) \mathrm{v}$ $\mathrm{n}=12(12.8 \%))$.

In MA+ compared to normoalbuminuric subjects; mean HbAlc levels were higher (10.7 (SD1.7) v 9.6 (1.4) \%, $\mathrm{p}<0.001)$, proportion of females was greater $(\mathrm{n}=54$ $\left.(58.1 \%) v \mathrm{n}=163(42.2 \%), \chi^{2}=7.6, \mathrm{p}=0.006\right)$, and mean height SDS was lower (0.0 (SD 1.0) $v 0.2($ SD 1.0), $\mathrm{p}=0.006)$. IGF-1 levels were lower in MA+ compared to normoalbuminuric subjects and these differences have been described in detail previously..$^{25}$

In subjects with LJM compared to those without LJM, mean ACR levels were higher (table 1 and fig 3B), but there was no difference in prevalence of MA (table 1). In subjects with LJM only, mean ACR levels were higher after the appearance of LJM compared to before (median 1.2 (range $0.4-102.6) v 0.8(0.2-181.9) \mathrm{mg} / \mathrm{mmol}, \mathrm{p}=0.003)$. From 95 MA+ subjects, 29 (30.5\%) had persistent and 66 (69.5\%) transient MA; however no difference was found in prevalence of LJM. Other differences between persistent and transient subjects have been detailed previously. ${ }^{25} 27$ 

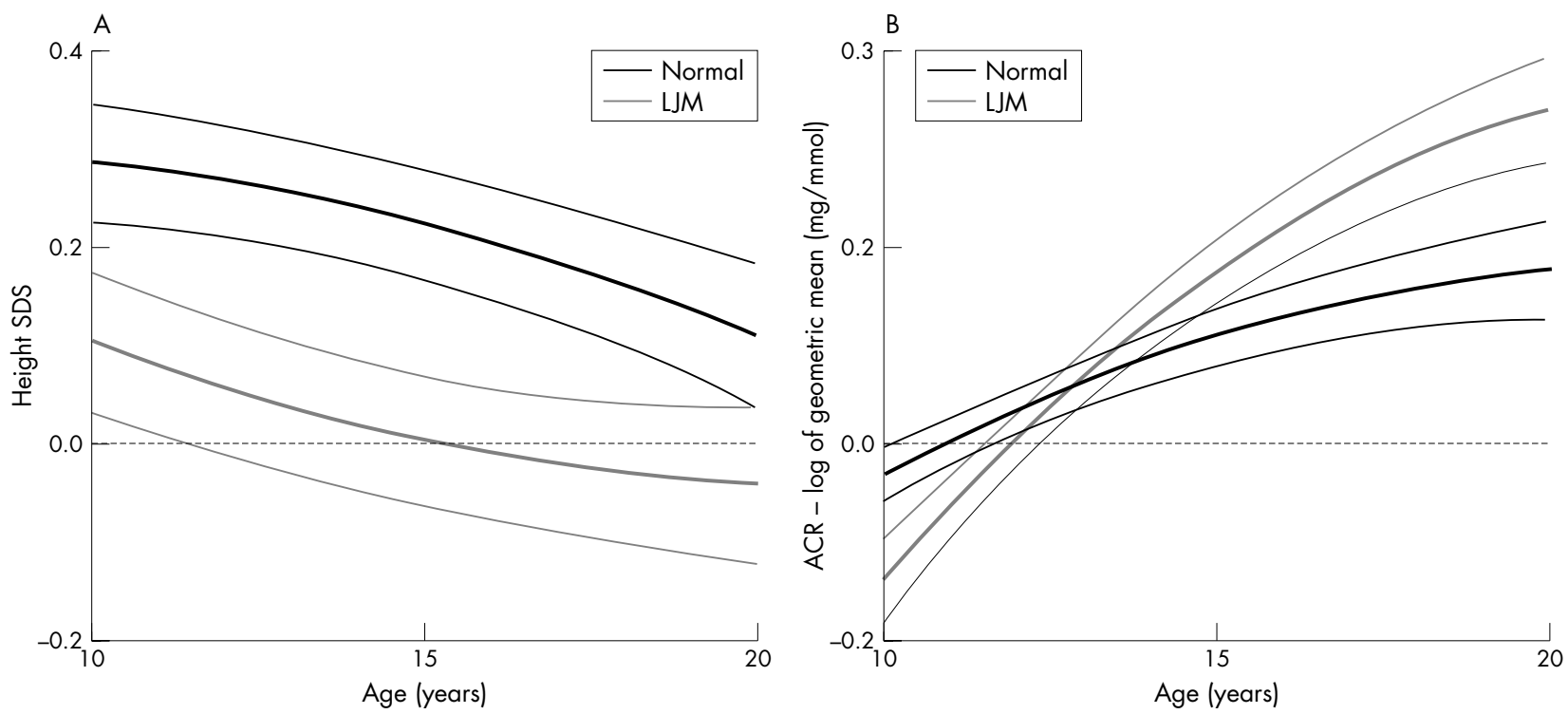

Figure 3 (A) Height SDS; (B) Urine albumin:creatinine ratio (ACR) plotted against age in subjects with and without limited joint mobility (LM). Figures derived from multi-level modelling, with thin lines representing \pm SEM (see text for details).

\section{Severity of LJM}

A total of $139(85.8 \%)$ had mild LJM and 23 (14.2\%) had moderate or severe LJM. Mean HbAlc and ACR levels were higher in those with severe/moderate LJM compared to those with mild LJM and no LJM ( $\mathrm{HbAl}_{\mathrm{c}}$ : mean 10.6 (SD 1.7) $v 9.3$ (0.9) v 9.1 (1.1) \%, p < 0.001; ACR: median 1.1 (range 0.4242.9) $v 1.0(0.4-107.8) v 0.9(0.1-81.2) \mathrm{mg} / \mathrm{mmol}, \mathrm{p}=0.02)$.

\section{Comparison of data after the onset of LJM}

In LJM+ subjects, using data only after the onset of LJM, compared to LJM- subjects: mean HbAlc (10.2 (SD 1.6) $v$ $9.6(1.4) \%, p=0.002$ ) and median ACR levels (1.2 (range 0.4-102.6) v $0.9(0.4-70.7) \mathrm{mg} / \mathrm{mmol}, \mathrm{p}=0.004)$ remained higher, and prevalence of MA was greater $(\mathrm{n}=37(23.7 \%) v$ $\left.\mathrm{n}=56(18.6 \%), \chi^{2}=3.2, \mathrm{p}=0.06\right)$. Furthermore height SDS $($ mean $-0.1($ SD 1.0) $v 0.2(1.1), \mathrm{p}<0.001)$ and IGF-1 SDS $(-1.0(1.5) v-0.5(1.4), \mathrm{p}=0.005)$ were lower.

\section{Relation between LJM, MA, and blood pressure}

Overall no difference existed in mean BP or BP SDS between subjects with and without LJM. In subjects with LJM after the development of LJM compared to before, mean systolic BP SDS was similar $(-1.2$ (SD 1.1) $v-1.1(1.1), \mathrm{p}=0.1)$; however mean diastolic BP SDS was greater (1.2 (1.3) $v 0.9$ (1.3), $\mathrm{p}=0.001$ )

Comparing those with $(\mathrm{MA}+)$ and without MA (MA-), mean systolic BP SDS was higher $(-1.1$ (SD 0.6) $v-0.9(0.7)$, $\mathrm{p}=0.04)$, however diastolic BP SDS was no different. When considering those with persistent MA compared to the MA - group, both systolic BP SDS $(-1.1 \quad(0.6) \quad v-0.9$ $(0.7), \mathrm{p}=0.05)$ and diastolic BP SDS (1.3 (0.6) $v$ 1.1 (0.6), $\mathrm{p}=0.05$ ) were higher. In subjects with MA, BP SDS was higher after compared to before the onset of MA (systolic: -0.8 (0.6) $v-1.2$ (0.7), $\mathrm{p}=0.01$; diastolic: 1.4 (1.0) $v 1.0$ $(0.9), \mathrm{p}=0.005)$.

\section{Determinants of risk for development of MA using Cox method}

Following the development of LJM, probability of developing MA was related to pubertal onset (that is, a 6.6-fold increased risk with pubertal onset), HbAlc (that is, a $20 \%$ increased risk for a $1 \%$ HbAlc rise), female sex (that is, a 2 -fold increased risk), and presence of LJM (a 1.9-fold increased risk if LJM was present) (table 2). Subjects with persistent MA were considered too few to analyse separately to transient subjects.

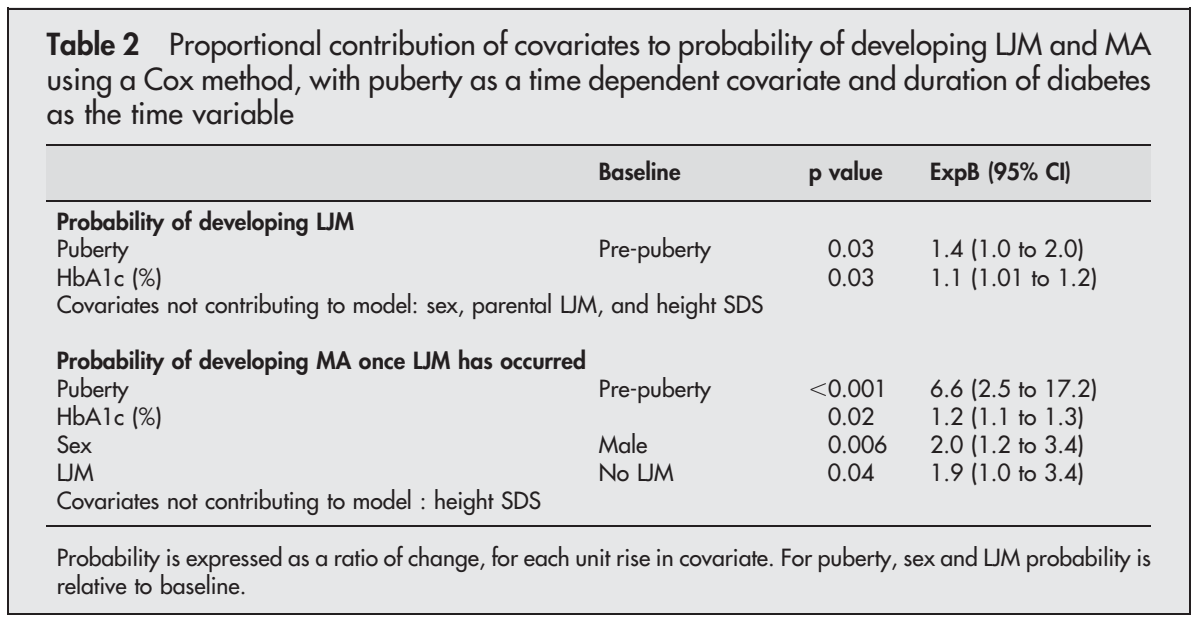




\section{DISCUSSION}

The ORPS is a prospective study of 479 TIDM children followed from diagnosis of diabetes through puberty. We describe the development of LJM in this cohort in relation to the development of MA. To reduce intra-observer variation in diagnosis of LJM, we restricted the definition to the finding of LJM during two or more annual assessments, as measured repeatedly by the same observer. Overall incidence was $35.1 \%$, which is comparable to previous reports, ${ }^{146}$ further validating this method of assessment.

We confirm longitudinal data from Rosenbloom et al indicating that risk of LJM is related to poor glycaemic control and duration of diabetes. ${ }^{6}{ }^{12}$ This is further confirmed by reduced incidence of LJM with improved glycaemic control $^{31}$ and in more contemporary cohorts. ${ }^{32}$ Chronic hyperglycaemia results in accumulation of advanced glycation end products which increase cross-linking in the subcutaneous tissues and formation of inflexible collagen. ${ }^{33}$ Other largely cross-sectional data are conflicting and show no such relation, which may reflect the use of only a single $\mathrm{HbAl}_{\mathrm{c}}$ value. $^{12} 48$

Two cross-sectional studies show LJM to be more prevalent in males, ${ }^{2}{ }^{10}$ while others show no gender difference. These conflicting results may reflect differences in duration of diabetes and glycaemic control between the sexes. The current study detected no difference between genders despite differences in HbAlc levels, and further follow up is required to detect any sexual dimorphism for risk of LJM.

The longitudinal nature of our study allows us to explore the temporal relation between development of LJM and microvascular disease. Subjects with LJM had higher urine albumin excretion, and presence of LJM was associated with a twofold increased risk of developing microalbuminuria, which also related to severity of LJM, confirming findings of the original study of Rosenbloom and colleagues. ${ }^{6}$ Thus our data confirm that the presence of LJM is a sign of impending microvascular disease. BP was increased in subjects who developed both MA and LJM, but was not predictive of either, suggesting that hypertension may influence progression of microvascular disease but is not involved in its initiation. This is in keeping with previous, more carefully controlled data from our group. ${ }^{34}$ Other risk factors for developing both microalbuminuria and LJM were found to be independent of $\mathrm{HbAlc}$, and suggest factors other than poor glycaemic control may be important.

Consistent with a cross-sectional study ${ }^{11}$ and longitudinal data that found appearance of LJM was more influenced by age than diabetes duration, ${ }^{19}$ we found that risk for developing LJM and microalbuminuria was associated with pubertal onset. Puberty is also a risk factor for retinopathy, ${ }^{35}$ and this suggests that the development of microvascular complications may be related to pubertal hormonal variables. Risk for diabetic complications may be associated with abnormalities of the GH-IGF-1 axis, particularly in females. ${ }^{36}$ We confirm previous findings that risk for microalbuminuria is increased in adolescent females. ${ }^{27}$ In the current study subjects with LJM had lower IGF-1 levels, and our group has recently shown that MA risk in the same cohort was related to lower IGF-1 levels ${ }^{25}$ and GH hypersecretion, ${ }^{37}$ particularly in females. In rodent models raised $\mathrm{GH}$ induces diabetic nephropathy-like changes, ${ }^{38}$ including increased cross-linking of collagen in the glomerulus. ${ }^{39}$

Previous data describe an association between LJM and attenuated growth ${ }^{4919}$ and between nephropathy risk and short stature. ${ }^{2021}$ The latter may relate to factors operating in utero to reduce birth weight and nephron number. ${ }^{21} 22$ However we found no differences in birth weight, suggesting that other mechanisms may be involved. Poor glycaemic control may lead to impaired growth in diabetic children; however the relation to puberty was independent of HbAlc levels, providing further evidence of the possible influence of the GH-IGF-1 axis on complications risk. The relation between short stature and nephropathy risk has been found in subjects diagnosed with TIDM after age 19 years, suggesting a genetic predisposition; ${ }^{22}$ however we found no evidence of familial clustering and further research is required to support this hypothesis.

In summary, this is the largest longitudinal study to describe the development of LJM and its association with microvascular disease. LJM is associated with puberty independent of glycaemic control and is predictive of microalbuminuria. LJM is easily assessed in a clinic setting; however specificity is poor and therefore is certainly not a substitute for screening by ophthalmology and urine albumin excretion. Thus pubertal subjects with poor glycaemic control, especially if female or with LJM, should all have formal assessment for microalbuminuria, hypertension, and retinopathy on an annual basis. Lower IGF-1 levels and attenuated growth in subjects with LJM were also found in relation to the development of microalbuminuria and may reflect shared underlying pathogenic mechanisms.

\section{ACKNOWLEDGEMENTS}

Rakesh Amin is a Novo Nordisk UK Research Foundation Fellow. The Oxford Regional Prospective Study is funded by Diabetes UK. We acknowledge The Juvenile Diabetes Research Foundation, the study field workers, the laboratory assistance of Angie Watts and Dot Harris, the Barts-Oxford Study field workers, and of paediatricians, physicians, and diabetes nurse specialists in the Oxford Region. We are indebted to Mrs Joan Hansen for skilled technical assistance, and JM Holly for analysis of IGF-1 levels.

\section{Authors' affiliations}

R Amin, T K Bahu, B Widmer, D B Dunger, University Department of Paediatrics, Addenbrookes Hospital, Cambridge, UK

R N Dalton, Children Nationwide Kidney Research Laboratory, Guy's Hospital, London, UK

Competing interests: none declared

Consent was obtained for publication of figure 1

Members of the Oxford Regional Prospective Study Steering Committee are: DB Dunger, RN Dalton, J Fuller, EAM Gale, H Keen, M Murphy, HAW Niel, CJ Schultz, RJ Young, and T Konopelska-Bahu

Members of the Oxford Regional Prospective Study are: JA Edge, John Radcliffe Hospital, Oxford; HAW Niel and David Matthews, The Oxford Centre for Diabetes, Endocrinology and Metabolism, The Churchill Hospital, Oxford; RAF Bell and A Taylor, Horton General Hospital, Banbury; A Mukhtar, BP O'Malley, BR Silk, and EH Smith, Kettering District Hospital, Kettering; RDM Scott, King Edward VII Hospital; FM Ackland, CJ Fox, and NK Griffin, Northampton General Hospital; N Mann, H Simpson, P Cove Smith, and M Pollitzer, Royal Berkshire Hospital, Reading; RS Brown and AH Knight, Stoke Mandeville Hospital, Aylesbury; JM Cowen and JC Pearce, Wexham Park Hospital, Slough

\section{REFERENCES}

1 Clarke CF, Piesowicz AT, Spathis GS. Limited joint mobility in children and adolescents with insulin dependent diabetes mellitus. Ann Rheum Dis 1990;49:236-7.

2 Duffin AC, Lam A, Kidd R, et al. Ultrasonography of plantar soft tissues thickness in young people with diabetes. Diabet Med 2002;19:1009-13

3 Garg SK, Chase HP, Marshall G, et al. Limited joint mobility in subjects with insulin dependent diabetes mellitus: relationship with eye and kidney complications. Arch Dis Child 1992;67:96-9.

4 Kakourou T, Dacou-Voutetakis C, Kavadias G, et al. Limited joint mobility and lipodystrophy in children and adolescents with insulin-dependent diabetes mellitus. Pediatr Dermatol 1994;11:310-14.

5 Larkin JG, Frier BM. Limited joint mobility and Dupuytren's contracture in diabetic, hypertensive, and normal populations. BMJ (Clin Res Ed) 1986;292:1494.

6 Rosenbloom AL, Silverstein JH, Lezotte DC, et al. Limited joint mobility in childhood diabetes mellitus indicates increased risk for microvascular disease. N Engl J Med 1981;305:191-4.

7 Arkkila PE, Kantola IM, Viikari RS, et al. Limited joint mobility is associated with the presence but does not predict the development of microvascular complications in type 1 diabetes. Diabet Med 1996;13:828-33. 
8 Huddle KR, Gill GV, Krige LP. Limited joint mobility in Black patients with type I diabetes mellitus. S Afr Med J 1983;64:579-81.

9 Grgic A, Rosenbloom AL, Weber FT, et al. Joint contracture-common manifestation of childhood diabetes mellitus. J Pediatr 1976;88(4 pt 1):584-8.

10 Frost $\mathrm{D}$, Beischer $\mathrm{W}$. Limited joint mobility in type 1 diabetic patients: associations with microangiopathy and subclinical macroangiopathy are different in men and women. Diabetes Care 2001;24:95-9.

11 Vukovic J, Dumic M, Radica A, et al. Risk factors for expression and progression of limited joint mobility in insulin-dependent childhood diabetes. Acta Diabetol 1996;33:15-18.

12 Silverstein JH, Gordon G, Pollock BH, et al. Long-term glycemic control influences the onset of limited joint mobility in type 1 diabetes. $J$ Pediatr $1998 ; 132: 944-7$

13 McCance DR, Crowe J, Quinn MJ, et al. Incidence of microvascular complications in type 1 diabetic subjects with limited joint mobility: a 10-year prospective study. Diabet Med 1993;10:807-10.

14 Montana E, Rozadilla A, Nolla JM, et al. Microalbuminuria is associated with limited joint mobility in type I diabetes mellitus. Ann Rheum Dis 1995;54:582-6.

15 Buckingham B, Perejda AJ, Sandborg C, et al. Skin, joint, and pulmonary changes in type I diabetes mellitus. Am J Dis Child 1986;140:420-3.

16 Silverstein JH, Fennell R, Donnelly W, et al. Correlates of biopsy-studied nephropathy in young patients with insulin-dependent diabetes mellitus. $J$ Pediatr 1985; 106:196-201.

17 Haitas $B$, Jones DB, Ting A, et al. Diabetic retinopathy and its association with limited joint mobility. Horm Metab Res 1986;18:765-7.

18 Rosenbloom AL, Malone JI, Yucha J, et al. Limited joint mobility and diabetic retinopathy demonstrated by fluorescein angiography. Eur J Pediatr 1984;141:163-4.

19 Rosenbloom AL, Silverstein JH, Lezotte DC, et al. Limited joint mobility in diabetes mellitus of childhood: natural history and relationship to growth impairment. J Pediatr 1982;101:874-8.

20 Chaturvedi N, Fuller J, Stephenson J. Short stature and diabetic nephropathy. BMJ 1995;310:1199.

21 Rossing P, Tarnow L, Nielson FS, et al. Short stature and diabetic nephropathy. BMJ 1995;310:296-7.

22 Hovind P, Tarnow L, Rossing P, et al. Predictors for the development of microalbuminuria and macroalbuminuria in patients with type 1 diabetes: inception cohort study. BMJ 2004;328:1105.

23 Penfold J, Chase HP, Marshall G, et al. Final adult height and its relationship to blood glucose control and microvascular complications in IDDM. Diabet Med 1995; 12:129-33.

24 Rossing P, Hougaard P, Borch-Johnsen K, et al. Predictors of mortality in insulin dependent diabetes: 10 year observational follow up study. BMJ 1996;313:779-84.
25 Amin R, Schultz C, Ong J, et al. Low IGF-I and elevated testosterone during puberty in subjects with type 1 diabetes developing microalbuminuria in comparison to normoalbuminuric control subjects: The Oxford Regional Prospective Study. Diabetes Care 2003;26:1456-61.

26 Feldmann B, Jehle PM, Mohan S, et al. Diabetic retinopathy is associated with decreased serum levels of free IGF-I and changes of IGF-binding proteins. Growth Horm IGF Res 2000; 10:53-9.

27 Schultz CJ, Konopelska-Bahu T, Dalton RN, et al. Microalbuminuria prevalence varies with age, sex, and puberty in children with type 1 diabetes followed from diagnosis in a longitudinal study. Oxford Regional Prospective Study Group. Diabetes Care 1999;22:495-502.

28 Davis JE, McDonald JM, Jarett L. A high-performance liquid chromatography method for hemoglobin Alc. Diabetes 1978:27:102-7.

29 Morrell DJ, Ray KP, Holder AT, et al. Somatomedin C/insulin-like growth factor 1: simplified purification procedure and biological activities of the purified growth factor. J Endocrinol 1986;110:151-8.

30 Cole TJ. The LMS method for constructing normalized growth standards. Eur J Clin Nutr 1990:44:45-60.

31 Lieberman L, Rosenbloom AL, Riley WJ, et al. Reduced skin thickness with pump administration of insulin. N Engl J Med 1980;303:940-1.

32 Infante J, Rosenbloom AL, Silverstein JH, et al. Changes in frequency and severity of limited joint mobility in children with type 1 diabetes mellitus between 1976-78 and 1998. J Pediatr 2001;138:33-7.

33 Monnier VM, Vishwanath V, Frank KE, et al. Relation between complications of type I diabetes mellitus and collagen-linked fluorescence. N Engl J Med 1986;314:403-8

34 Schultz CJ, Neil HA, Dalton RN, et al. Blood pressure does not rise before the onset of microalbuminuria in children followed from diagnosis of type 1 diabetes. Oxford Regional Prospective Study Group. Diabetes Care 2001;24:555-60.

35 Danne T, Kordonouri O, Enders I, et al. Factors modifying the effect of hyperglycemia on the development of retinopathy in adolescents with diabetes. Results of the Berlin Retinopathy Study. Horm Res 1998;50(suppl 1):28-32.

36 Edge JA, Dunger DB, Matthews DR, et al. Increased overnight growth hormone concentrations in diabetic compared with normal adolescents. J Clin Endocrinol Metab 1990;71:1356-62.

37 Amin R, Williams RM, Frystyk J, et al. Increasing urine albumin excretion is associated with growth hormone hypersecretion and reduced clearance of insulin in adolescents and young adults with type 1 diabetes: The Oxford Regional Prospective Study. Clin Endocrinol (Oxf) 2005;62:137-44.

38 Flyvbjerg A. Putative pathophysiological role of growth factors and cytokines in experimental diabetic kidney disease. Diabetologia 2000;43:1205-23.

39 LePape A, Guitton J, Muh J. Modifications of glomerular basement membrane cross-links in experimental diabetic rats. Biochem Biophys Res Commun $1981 ; 100: 1214-21$. 